\title{
USO DA SEMENTE DE AÇAÍ COMO ALTERNATIVA ENERGÉTICA NA INDÚSTRIA DE BENEFICIAMENTO DE POLPA DE AÇAÍ
}

\author{
Mohara Silva Passinho ${ }^{1}$, Diérfano de Lima Barbosa ${ }^{2}$, Marcelo José Raiol Souza ${ }^{3}$, \\ Nilza Martins de Queiroz Xavier ${ }^{4}$ \\ ${ }^{1}$ Engenheira Ambiental pela Universidade do Estado do Pará (UEPA), Belem-PA, \\ Brasil. \\ ${ }^{2}$ Engenheiro Ambiental pela Universidade do Estado do Pará (UEPA), Belém-PA, \\ Brasil. \\ ${ }^{3}$ Professor Doutor do curso de Engenharias Florestal e Ambiental da UEPA, Belém- \\ PA, Brasil.mraiol@yahoo.com.br \\ ${ }^{4}$ Engenheira Ambiental pela Universidade do Estado do Pará (UEPA), Belem-PA, \\ Brasil.
}

\section{Recebido em: 06/04/2019 - Aprovado em: 10/06/2019 - Publicado em: 30/06/2019 DOI: 10.18677/EnciBio 2019A131}

\begin{abstract}
RESUMO
O desenvolvimento industrial trouxe consigo a geração de resíduos, que demandam cada vez mais soluções para acondicionamento e destinação final. A biomassa corresponde a uma energia renovável, ela é encontrada principalmente nos resíduos, em diversas atividades em especial nas indústrias madeireira e alimentícia. Dentre as indústrias alimentícias, a indústria de beneficiamento de açaí, teve um grande crescimento, impulsionado pela enorme procura de derivados do produto. As indústrias de açaí tem como principal resíduo sólido do processo produtivo as sementes de açaí, geradas em grande quantidade e que atualmente são aproveitadas para diversas finalidades dentre elas a geração de energia térmica. Esse processo ocorre por meio de combustão direta na caldeira flamotubular que fornece energia térmica para os pasteurizadores e tanque de mistura. Para o presente estudo foi realizada coleta e análise imediata do teor de umidade das sementes, balanço de massa e energia da caldeira e mix energético entre os dois combustíveis, com intuito de apontar se seria mais vantajosa a substituição parcial ou total da lenha pelas sementes de açaí. Os resultados mostraram que as sementes de açaí coletadas tem desempenho energético superior ao da lenha, possuem um baixo teor de umidade (12\%), poder calorífico inferior de 17896,42 $\mathrm{kJ} / \mathrm{kg}$ e liberam entalpia de saída de vapor de $3490,21 \mathrm{KJ} / \mathrm{kg}$, podendo substituir o combustível lenha integralmente, fornecendo maior quantidade de energia para os pasteurizadores e tanque de mistura, dessa forma auxiliando no processo produtivo e interrompendo os gastos com a aquisição de lenha.
\end{abstract}

PALAVRAS-CHAVE: açaí, biomassa, caldeira

\section{USE OF SEEDS OF AÇAÍ AS AN ENERGY ALTERNATIVE IN THE AÇAí POWDER BENEFIT INDUSTRY}

\section{ABSTRACT}

The industrial development brought industrial waste, that demand each day more solutions to the packaging and final destination. The biomass correspond to a 
renewable energy, it is found principally in the industrial waste, in several activities, especially in the lumberyard and food industry. Among the food industry, the industry of processing of açaí, had a big grownth, impelled by the big search of by-product of this product. The açaí industry has as principal residue of the constructive process the seeds of açaí, generated in a big amount and that currently are used to several purpose, among it the generation of thermic energy. This process happens through direct combustion in a flamotubular boiler that provides thermical energy to the pasteurisers and mixing tanks. In this paper was performed a immediate gathering and analysis from the humidity content of the seeds, check of mass and energy of the boiler and mix energetic between two fuels, with the intention of point out if it would have more advantage in partial replacement or complete replacement from the firewood to the seeds of açaí. The outcome had shown that the colected seeds of açaí has a higher energy performance than firewood, have low humidity content $(12 \%)$, lower calorific power of $17896,42 \mathrm{~kJ} / \mathrm{kg}$ and release enthalpy of steam output of $3490,21 \mathrm{KJ} / \mathrm{kg}$, can whole replace the firewood fuel, providing bigger amount of energy to the pasteurisers and mixing tanks, in doing that support the productive process and interrupts the wastes with the acquisition of firewood.

KEYWORDS: açaí, biomass, boiler

\section{INTRODUÇÃO}

Um dos maiores desafios para se alcançar a sustentabilidade está em solucionar a questão da poluição e da escassez dos recursos naturais, embora pareçam conflitantes dentro da sociedade capitalista, a redução do impacto ambiental tornou-se uma exigência para as empresas que desejam continuar atuando no mercado, tanto nacional quanto internacional, pois produções mais limpas agregam valor ao produto fabricado tornando-o mais competitivo num mercado cada vez mais exigente no aspecto ambiental e social (OLIVEIRA NETO et al., 2015).

Dentro deste contexto o aproveitamento de resíduos sólidos lignocelulósicos ou biomassa lignocelúlosica para geração de energia térmica pelas indústrias vem ganhando destaque no cenário mundial, pois além de reduzir os impactos causados pela disposição incorreta na natureza, trás benefícios energéticos com sua transformação em energia útil com uso de tecnologias como combustão direta, gaseificação, torrefação, briquetagem e peletização (ALMADA et al., 2017).

Sabe-se que muitas indústrias utilizam o processo de combustão como um dos principais meios de geração de energia térmica, e como consequências dessa geração de energia útil podem surgir impactos ambientais negativos. Seus efeitos no meio ambiente estão ligados a problemas de ordem política, social e econômica (SANTOS; LIMA, 2016). Sob essa perspectiva, as empresas enxergaram nos resíduos lignocelulósicos, a oportunidade de uso na geração de energia térmica como forma de suprir as necessidades energéticas do próprio processo produtivo. Desta maneira este tipo de biomassa surge como oportunidade, devido ser fonte de energia renovável e disponível em abundância.

Diversos estudos e técnicas aplicados as mais variadas transformações de resíduos em energia são desenvolvidos, em especial na indústria alimentícia, dentre elas as análises de consumo energético e eficiência no processamento de alimentos que envolvem aplicação dos princípios científicos e de engenharia como física, química, termodinâmica, transferência de calor e mecânica dos fluidos (ÇENGEL; BOLES, 2015). Esse é o caso das sementes de açaí, resultado da produção de 
polpa de açaí, alimento que sempre esteve presente no cotidiano da população amazônica, tendo seu beneficamento realizado de maneira tradicional nas chamadas batedeiras de açaí.

Devido a forte demanda externa por polpa de açaí, a cadeia produtiva ganhou conformação com o processamento industrial em escala completamente diferente daquela realizada nas batedeiras das quitandas ou baiúcas (SANTANA et al., 2006). Esse grande crescimento industrial veio acompanhado pela constante geração de resíduos, principalmente de sementes de açaí, que devido o elevado poder calorífico (RENDEIRO et al., 2008) comprovam serem grandes aliadas na geração de energia térmica. Isso posto, o presente trabalho teve como objetivo estimar a viabilidade energética do uso da biomassa semente de açaí na matriz energética de uma indústria de beneficiamento de polpa de açaí localizada no município de CastanhalPA.

\section{MATERIAIS E MÉTODOS}

O estudo foi realizado em uma indústria de produção de polpa de açaí, localizada no município de Castanhal-PA, que utiliza, principalmente, lenha em uma caldeira do tipo flamotubular horizontal (figura 1), para geração de energia térmica à ser utilizada no processo produtivo. Inicialmente foram coletadas informações sobre o processo e também amostras de semente de açaí, dispostas em um ponto específico do parque fabril chamado de silo de armazenagem, com objetivo de determinação do teor de umidade. Por fim foi realizada um tratamento termodinâmico com as informações obtidas.

\section{Coleta de informações}

Foram coletadas informações referentes à quantidade e procedência da lenha utilizada para queima na caldeira e de resíduos de semente de açaí gerados diariamente na indústria em análise. A lenha utilizada para queima é proveniente de serrarias, localizadas próximas a indústria, já o consumo desse combustível foi estimado em $0,11 \mathrm{~m}^{3} / \mathrm{h}$. As informações técnicas da caldeira, necessárias para o tratamento termodinâmico, foram obtidas no prontuário da caldeira, disponibilizada pela indústria. Essas informações estão apresentadas no quadro 1.

A quantidade de sementes de açaí geradas pela indústria, foi estimada, considerando a quantidade de 3.000 latas de frutos processadas diariamente. De acordo com EMBRAPA (2005) cada lata possui $14 \mathrm{~kg}$ de frutos, em que a semente representa $85 \%$ do peso total, dessa forma é possível estimar que a massa de sementes produzida por dia é de $35.700 \mathrm{~kg}$ e portanto são $1.487,5 \mathrm{~kg}$ de sementes de açaí geradas por hora. Grande parte destas sementes são destinadas para as olarias da região e o restante fica na indústria.

QUADRO 1 - Informações técnicas da caldeira

\begin{tabular}{|l|l|}
\hline Item & Característica \\
\hline Modelo & Horizontal Flamotubular \\
\hline Produção de vapor- c/água a $\left(20^{\circ} \mathrm{C}\right)$ & $250 \mathrm{~kg} / \mathrm{h}$ \\
\hline Produção de vapor- c/água a $\left(80^{\circ} \mathrm{C}\right)$ & $280 \mathrm{~kg} / \mathrm{h}$ \\
\hline Consumo de combustível (lenha) & $0,11 \mathrm{~m}^{3} / \mathrm{h}$ \\
\hline Pressão de Trabalho & $5 \mathrm{kgf} / \mathrm{cm}^{2}$ \\
\hline Estado do vapor produzido & saturado \\
\hline Temperatura da água de entrada na caldeira & $35^{\circ} \mathrm{C}$ \\
\hline
\end{tabular}


O fluxograma do processo produtivo é apresentado na figura 2, onde destacase os equipamentos que utilizam a energia térmica produzida pela caldeira. Vale salientar que a caldeira é circundada esquematicamente por uma linha imaginária que define uma região do espaço onde serão conduzidas as análises termodinâmicas.

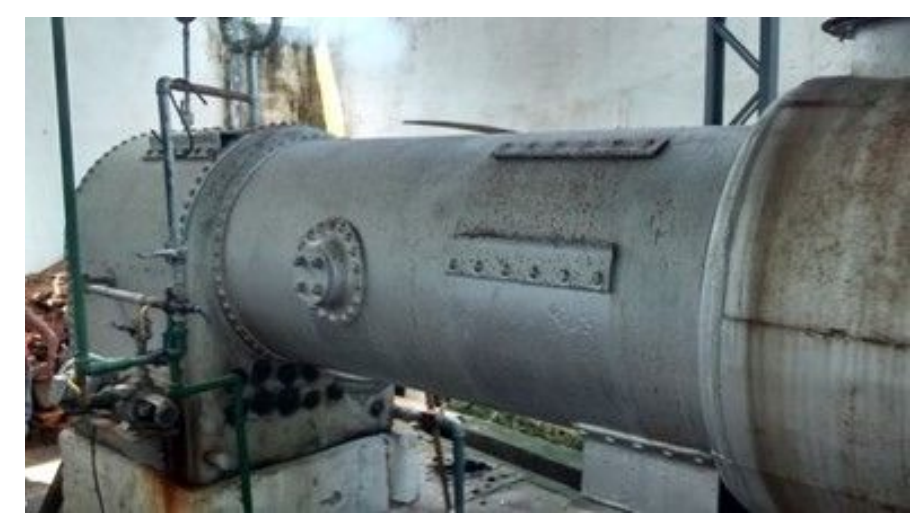

FIGURA 1 - Caldeira Flamotubular horizontal

Fonte: Autores (2016)

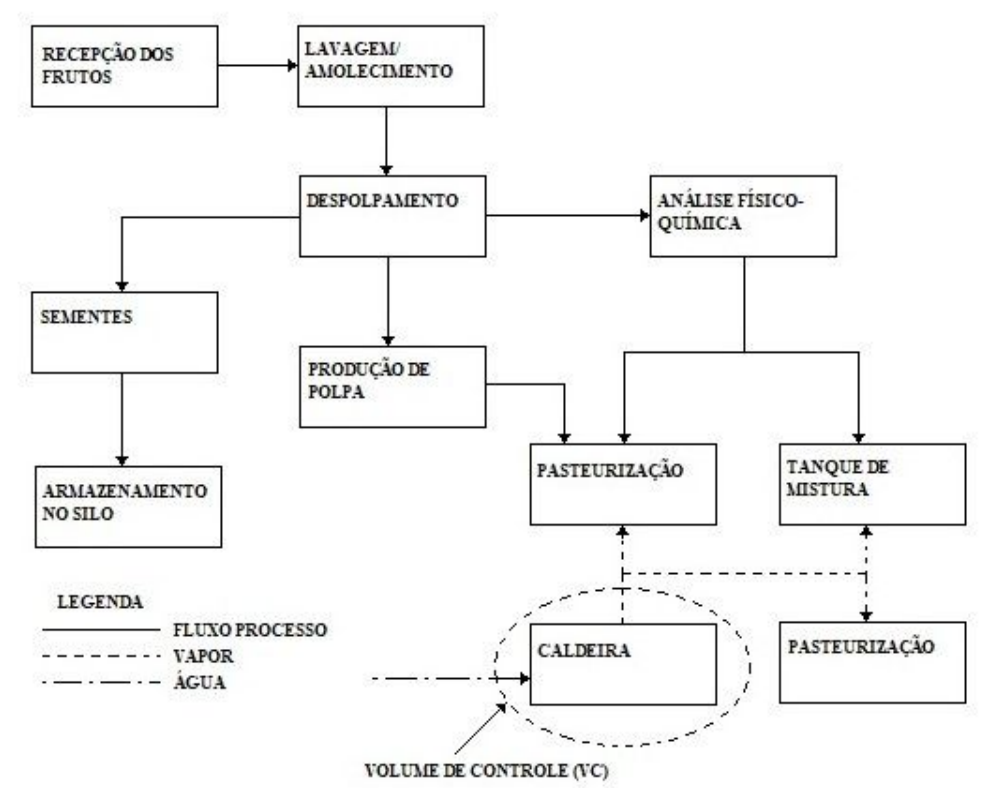

FIGURA 2 - Fluxograma simplificado do processo produtivo incluindo etapa de geração de vapor utilizado na análise.

Fonte: Autores (2016)

\section{Determinação da umidade}

As análises das amostras de semente de açaí foram realizadas no Laboratório de Madeira da Universidade do Estado do Pará, com auxílio da metodologia apresentada no trabalho de Almada et al. (2017). O objetivo das análises foi determinar o teor de umidade médio de seis amostras retiradas do silo de armazenamento. De acordo com a norma foram pesadas aproximadamente 500 gramas de cada amostra e posteriormente colocadas em estufa a temperatura de $105^{\circ} \mathrm{C}$, em seguida as mesmas foram levadas para um dessecador e depois foram novamente pesadas em uma balança de precisão, para verificar a variação da 
umidade. Após estas etapas, os valores encontrados foram substituídos na equação (1), que determina o teor de umidade. Por fim foi calculada a média aritmética de seis amostras analisadas.

$\mathrm{Tu}=100(\mathrm{mBo}-\mathrm{mBf}) / \mathrm{mBo}$

Onde: Tu é o teor de umidade em \%, mBo a massa de biomassa inicial em gramas; $\mathrm{mBf}$ a massa de biomassa final em gramas.

\section{Tratamento termodinâmico}

\section{Determinação da vazão mássica de combustível}

A seguir são apresentados os cálculos energéticos realizados tanto para as sementes de açaí quanto para a lenha. Por se tratar de um modelo antigo de caldeira, as mediçoes diretas de consumo de combustível ficaram inviáveis. Desta maneira foi assumido que a caldeira consome $0,11 \mathrm{~m}^{3} / \mathrm{h}$ de lenha, dado obtido no quadro 1. A espécie de lenha mais utilizada para queima é a Manilkara huberi (maçaranduba). Para o cálculo da massa de lenha que entra por hora na caldeira é necessário conhecer a massa específica aparente, cujo o valor para a espécie considerada é $850 \mathrm{~kg} / \mathrm{m}^{3}$ (REMADE, 2016). Com essas informações foi calculada a vazão mássica da biomassa de acordo com a definição da massa específica aparente.

$\rho=m / V$

Onde: $\rho$ é a massa específica em $\mathrm{kg} / \mathrm{m}^{3}, \mathrm{~m}$ a massa em $\mathrm{kg}, \mathrm{V}$ o volume em $\mathrm{m}^{3}$

\section{Poder Calorífico Superior e Inferior}

Os cálculos foram realizados utilizando os resultados de análise elementar dos combustíveis lenha e semente de açaí disposto na literatura especializada (RENDEIRO et al., 2008; NOBRE et al., 2015). A determinação do Poder Calorífico Superior e Inferior das biomassas, foram realizadas utilizando as equações (3) e (4) (LOO; KOPPEJAN, 2008). A composição elementar de semente de açaí e lenha são apresentados na tabela 1 .

TABELA 1 - Composição elementar da lenha de Manilkara huberi (Maçaranduba) e de semente de açaí.

\begin{tabular}{|l|c|c|}
\hline \multirow{2}{*}{$\begin{array}{l}\text { Elementos } \\
\text { químicos }\end{array}$} & \multicolumn{2}{|c|}{ Quantidade (\%) } \\
\cline { 2 - 3 } & $\begin{array}{l}\text { Lenha (Manilkara huberi) } \\
\text { (NOBRE et al. 2015) }\end{array}$ & $\begin{array}{l}\text { Semente } \\
\text { (RENDEIRO et al. 2008) }\end{array}$ \\
\hline C (Carbono) & 49,54 & 46,0 \\
\hline H (Hidrogênio) & 6,31 & 6,0 \\
\hline N (Nitrogênio) & 0,67 & 0,8 \\
\hline S (Enxofre) & 0,01 & - \\
\hline O (Oxigênio) & 43,45 & 46 \\
\hline
\end{tabular}

As quantidades dos elementos químicos presentes na lenha e semente de açai foram substituídas na equação (3) que calcula Poder Calorífico Superior (PCS). O valor do PCS é então substituido na equação (4) e juntamente com o valor da umidade calcula-se o pode calorífico inferior $(\mathrm{PCl})$. $\mathrm{O}$ valor de umidade das 
sementes de açaí foi obtido no laboratório enquanto para a lenha foi utilizado o valor de $40 \%$, valor fornecido pelos operadores da caldeira.

PCS $=-8419,7+479,3 . \mathrm{C}+667,6 . \mathrm{H}+58,8 . \mathrm{O}-1207,7 . \mathrm{S}$

$P C I=P C S .(1-w / 100)-2,447 .(w / 100)-2,447 .(H / 100) \cdot 9,01(1-w / 100)$

A quantidade de calor liberada no interior da caldeira no intervalo de tempo através da queima do combustível pode ser obtida pela definição de poder calorífico. $\mathrm{Na}$ equação (5) foi utilizado o poder calorífico inferior em virtude do mesmo considerar a quantidade de umidade presente no material combustível, ou seja, representa mais fielmente as condições reais de operação da caldeira.

$Q_{C}=P C I . m$

Onde: $Q_{c}$ é o calor fornecido pela queima de combustível em $\mathrm{KJ} / \mathrm{h}, \mathrm{PCl}$ o poder calorífico inferior em $\mathrm{KJ} / \mathrm{Kg}$, $\mathrm{m}$ a vazão mássica do combustível em $\mathrm{Kg} / \mathrm{h}$.

\section{Rendimento da caldeira}

Segundo Vakkilainen (2017) caldeiras flamotubulares possuem um baixo rendimento térmico, isso pode ser explicado pela maior dificuldade de manutenção da caldeira, projeto termico pouco eficiente, e também por essas caldeiras apresentarem sérios problemas de incrustação e depósito no lado dos gases no decorrer de seu tempo de vida útil. Associado a isso, foi levado em consideração o fato da caldeira da empresa ser bastante antiga. Para efeito de cálculos, foi considerada a hipótese de que a caldeira possui um rendimento mínimo de aproximadamente $50 \%$, valores menores não foram considerados pois seria inviável para a empresa ter em seu processo produtivo uma caldeira com rendimento térmico muito baixo. A partir do rendimento da caldeira e a energia liberada na combustão da lenha ou semente de açaí é possível estimar a energia térmica que efetivamente é produzida pelo equipamento, ou seja, quantidade e qualidade de vapor produzido. Tal estimativa é calculada pela definição de rendimento.

$\eta=Q / Q_{c}$

Onde: $\eta$ é o rendimento da caldeira, $Q$ o calor efetivo absorvido pela caldeira em $\mathrm{KJ} / \mathrm{Kg}$.

\section{Conservação da massa}

As transformações energéticas necessárias para a viabilidade do processo ocorrem no interior do equipamento caldeira. Para esta análise é necessário definir um volume de controle (VC) (figura 2), que é uma região do espaço escolhida convenientemente para análises pertinentes, no presente trabalho o VC circunda a caldeira e mostra claramente que existe uma única entrada de água e uma única saída de vapor. Segundo Çengel e Boles (2015) a equação da conservação da massa para um volume de controle (VC) se apresenta como:

$\mathrm{dm}_{\mathrm{vd}} / \mathrm{dt}+\sum \mathrm{m}_{\mathrm{s}}-\sum \mathrm{m}_{\mathrm{e}}=0$ 
Onde $\mathrm{dm}_{\mathrm{Vc}} / \mathrm{dt}$ é a taxa de variação de massa no interior do $\mathrm{VC}, \mathrm{m}_{\mathrm{s}}$ é o fluxo mássico de saída do VC, $m_{e}$ é o fluxo mássico de entrada no VC.

No presente trabalho será considerado regime permanente, ou seja, as propriedades termofísicas do vapor produzido pela caldeira e a água de alimentação da caldeira não variam como o tempo. Além disso, não há variação de massa no interior de VC, logo a taxa de variação de massa é zero, e a equação (7) fica:

$$
\sum \mathrm{m}_{\mathrm{s}}-\sum \mathrm{m}_{\mathrm{e}}=0
$$

Portanto o fluxo de entrada de massa de água $m_{e}$ será igual ao fluxo de saída de vapor $m_{s}$ que de acordo com o valor do quadro 1 é de $250 \mathrm{~kg} / \mathrm{h}$.

\section{Conservação de energia - 1a lei da Termodinâmica}

A expressão geral da Primeira Lei da Termodinâmica, para um VC é dada pela equação (9) (ÇENGEL; BOLES, 2015):

$$
Q_{v c}+\sum m_{e}\left(h_{e}+V_{e}^{2} / 2+g Z_{e}\right)=d E_{v c} / d t+\sum m_{s}\left(h_{s}+V_{s}^{2} / 2+g Z_{s}\right)+W_{v c}
$$

Onde: $Q_{v c}$ é a taxa de transferência de calor que entra ou sai do $V C, h_{e}$ a entalpia de entrada no $\mathrm{VC}, \mathrm{V}_{\mathrm{e}}$ a velocidade de entrada no $\mathrm{VC}, \mathrm{g}$ a aceleração da gravidade, $\mathrm{Z}_{\mathrm{e}}$ a cota na entrada no $\mathrm{VC}, \mathrm{dE} \mathrm{Vc}_{\mathrm{c}} / \mathrm{dt}$ a taxa de variação da energia no interior de $\mathrm{VC}$, $\mathrm{h}_{\mathrm{s}}$ a entalpia de saída do VC, $\mathrm{V}_{\mathrm{s}}$ a velocidade de saída do $\mathrm{VC}, \mathrm{Z}_{\mathrm{s}}$ a cota na saída do VC e Wvc a taxa de transferência de trabalho que entra ou sai do VC.

As energias cinética e potencial presentes na equação (9) são desprezíveis comparadas as trocas de energia térmica que ocorrem no interior da caldeira. Além disso, o regime de escoamento é considerado permanente e não há taxa de trabalho atravessando o VC. Portanto, a equação (9) fica:

$\mathrm{Q}_{\mathrm{vc}}+\sum \mathrm{m}_{\mathrm{e}} \mathrm{h}_{\mathrm{e}}=\sum \mathrm{m}_{\mathrm{s}} \mathrm{h}_{\mathrm{s}}$

Como no processo em análise só existe uma entrada de água e uma saída de vapor da caldeira, a equação (10) fica reduzida a:

$Q_{v c}+m_{e} h_{e}=m_{s} h_{s}$

Pela lei da conservação da massa, a vazão mássica de entrada é igual a vazão mássica de saída, ou seja, $m_{e}=m_{s}=m_{v}$. Logo a equação (11) fica:

$Q_{v c}+m_{v} h_{e}=m_{v} h_{s}$

\section{Entalpias de entrada e saída}

O cálculo do valor da entalpia da água de entrada no sistema foi realizado com o Software STEAM TABLE desenvolvido por FIGENER S/A (2000). Para esse 
cálculo foram considerados os valores de Pressão $\left(5 \mathrm{kgf} / \mathrm{cm}^{2}\right)$ e Temperatura $\left(35^{\circ} \mathrm{C}\right)$ fornecidos no quadro 1, dessa forma foi possível calcular o valor da entalpia de saída do sistema, utilizando a equação (12).

\section{Mix energético}

A principal proposta do presente trabalho é analisar energeticamente o uso de semente de açaí para geração de energia, entretanto, não se pode susbstituir cem por cento a queima da lenha pelo caroço de açaí, pois existem outras variáveis operacionais como: ar de combustão, geração de cinzas, formas de alimentação do combustível etc. que podem causar flutuações na geração de vapor para o processo. Para contornar este tipo de problema são estudadas, inicialmente, misturas (mix) entre a lenha e a semente de açaí. Assim, a substituição se dará de forma gradativa, permitindo tempo para o processo se adptar as novas configurações de queima. No mix energético se observa que a quantidade total de calor liberado pela queima é dada pela soma das quantidades de calor fornecidas pela queima de lenha e de sementes de açaí, de acordo com a seguinte equação.

$Q_{M I X}=Q_{R S}+Q_{R L}$

Onde: $Q_{M I X}$ é a quantidade de calor liberado pela mistura em $\mathrm{KJ} / \mathrm{h}, \mathrm{Q}_{\mathrm{RS}}$ a quantidade real de calor liberado pelas sementes de açaí em $\mathrm{KJ} / \mathrm{h}, \mathrm{Q}_{\mathrm{RL}}$ a quantidade real de calor liberado pela lenha em $\mathrm{KJ} / \mathrm{h}$.

Substituindo as equações (5), (6) e (12) em (13); observando que $Q_{M I X}=Q_{V c}$ $=\mathrm{Q}$ e após rearranjos encontra-se:

$h_{s}=\eta\left(m_{R S} P C l_{R S}+m_{R L} P C l_{R L}\right) / m_{v}+h_{e}$

Onde: $h_{\mathrm{s}}$ assume o valor da entalpia de saída de vapor, $m_{\mathrm{v}}$ o fluxo de água e vapor, $\mathrm{PCl}_{\mathrm{RS}}$ o poder calorífico inferior da semente de açaí, $\mathrm{PCl} \mathrm{RL}_{\mathrm{RL}}$ o poder calorífico inferior da lenha, $m_{R S}$ a vazão mássica da biomassa semente, $m_{R L}$ a vazão mássica da lenha, $h_{e}$ a entalpia de entrada de água.

As quantidades de lenha e biomassa caroço de açaí utilizadas no mix energético analisado são apresentadas na tabela 2.

TABELA 2 - Quantidades de combustíveis utilizado no mix energético

\begin{tabular}{|c|c|c|c|}
\hline \multicolumn{2}{|c|}{ Semente de açaí } & \multicolumn{2}{c|}{ Lenha } \\
\hline $\begin{array}{c}\text { Percentagem no } \\
\text { mix (\%) }\end{array}$ & Massa (Kg) & $\begin{array}{c}\text { Percentagem } \\
\text { no mix (\%) }\end{array}$ & Massa (Kg) \\
\hline 90 & 84,15 & 10 & 9,35 \\
\hline 80 & 74,5 & 20 & 18,7 \\
\hline 70 & 65,45 & 30 & 28,05 \\
\hline 60 & 56,1 & 40 & 37,04 \\
\hline 50 & 46,75 & 50 & 46,75 \\
\hline 40 & 37,4 & 60 & 56,1 \\
\hline 30 & 28,05 & 70 & 65,45 \\
\hline 20 & 18,7 & 80 & 74,8 \\
\hline 10 & 9,35 & 90 & 84,15 \\
\hline
\end{tabular}




\section{RESULTADOS E DISCUSSÕES}

Os modelos matemáticos apresentados foram implementadas no Software Excel 2010 e seus resultados são mostrados nos parágrafos seguintes. O cálculo da vazão mássica de entrada na caldeira, considerando o volume e a massa específica aparente da lenha, apresentou valor de 93,5 kg/h. Esse mesmo valor é considerado para as sementes de açaí, pois uma quantidade de sementes superior à estipulada, implicaria em problemas tais como entupimento da caldeira. Logo a vazão mássica de entrada no sistema será a mesma tanto para a lenha quanto para as sementes de açaí.

O cálculo do Poder Calorífico Superior da lenha $(22080,16 \mathrm{~kJ} / \mathrm{kg})$ foi maior que o da semente de açaí $(20338,5 \mathrm{~kJ} / \mathrm{kg})$. Isso ocorreu pelas diferenças na composição química elementar entre os dois combustíveis, pois a lenha apresenta percentuais maiores dos elementos Hidrogênio $(6,31 \%)$ e principalmente Carbono $(49,54 \%)$. De acordo com o trabalho de Rendeiro et al. (2008) há uma tendência de maiores valores de PCS estarem associados a maiores valores percentuais de hidrogênio $(H)$ e carbono $(C)$, enquanto o oxigênio embora seja um elemento essencial para promover a combustão do material não contribui para o aumento do PCS, pois altos valores de oxigênio diminuem o poder calorífico. Esse fato se aplica as sementes de açaí que possuem maior quantidade de oxigênio em sua composição $(46 \%)$ em relação à quantidade de oxigênio encontrado na lenha de maçaranduba $(43,45 \%)$.

O resultado do $\mathrm{PCl}$ das sementes foi maior $(17896,42 \mathrm{~kJ} / \mathrm{kg})$ do que da lenha $(13246,28 \mathrm{~kJ} / \mathrm{kg})$. Embora a lenha tenha apresentado PCS maior, o cálculo do PCI considera o valor do PCS em conjunto com o teor de umidade e percentual de hidrogênio. $O$ fator umidade foi determinante visto que o percentual de umidade encontrado nas sementes de açaí da indústria foi de (12\%) enquanto da lenha (40\%). Ou seja, quanto maior o teor de umidade, menor será o PCl, implicando que menor energia poderá ser extraída da biomassa (RENDEIRO et al., 2008).

Com o uso do software STEAM TABLE e dos valores da pressão $\left(5 \mathrm{kgf} / \mathrm{cm}^{2}\right)$ e temperatura $\left(35^{\circ} \mathrm{C}\right)$, foi obtido o valor de entalpia de entrada da água $\left(\mathrm{h}_{\mathrm{e}}\right)$ que corresponde a $147 \mathrm{~kJ} / \mathrm{kg}$. Após a realização do balanço de massa e energia foi possível observar que aumento da eficiência da caldeira, proporciona maior liberação de energia térmica, representada pelo valor de entalpia de saída do vapor $\left(h_{s}\right)$. Para essa simulação foram estimados valores percentuais de eficiência da caldeira, partindo de 50 até $80 \%$ para a lenha e semente de açaí e os resultados são mostrados na tabela 3.

TABELA 3 - Entalpia de saída do vapor produzido por lenha e por semente de açaí em $(\mathrm{kJ} / \mathrm{kg})$ considerando diferentes rendimentos em (\%)

\begin{tabular}{|c|c|c|}
\hline Rendimento (\%) & \multicolumn{2}{|c|}{ Entalpias de saída do vapor (KJ/kg) } \\
\cline { 2 - 3 } & Lenha & Semente de açaí \\
\hline 50 & 2621,48 & 3490,21 \\
\hline 60 & 3116,39 & 4158,89 \\
\hline 70 & 3611,35 & 4827,56 \\
\hline 80 & 4106,26 & 5496,19 \\
\hline
\end{tabular}

O valor de $2621,48 \mathrm{KJ} / \mathrm{kg}$ corresponde à entalpia de saída do vapor considerando a caldeira operando com $50 \%$ de rendimento, ou seja, rendimento mínimo, pois a caldeira opera em condições de pouca manutenção e muito tempo de 
uso. Esse valor representa a quantidade de energia que está sendo liberada atualmente pela caldeira através da queima de lenha. Por outro lado, se o combustível da caldeira for substituindo totalmente pela semente de açaí nas mesmas condições operacionais, observa-se uma liberação de energia de 3490,21 $\mathrm{KJ} / \mathrm{kg}$, ou seja, uma quantidade superior a observada atualmente com a queima de lenha.

De acordo com Vakkilainen (2017) o rendimento total que pode ser obtido na caldeira do tipo aquatubular é maior que a caldeira do tipo flamotubular, pois nessa última é impossível superar valores de $75 \%$ a $78 \%$ nas melhores condições de limpeza. Analisando ainda a tabela 3 constata-se que a caldeira operando com $70 \%$ de rendimento produz energia liberada pela queima da lenha inferior a semente de açaí, ou seja, entalpia de $3611,35 \mathrm{KJ} / \mathrm{kg}$ liberada pela lenha contra 4827,56 KJ/kg da semente de açaí. Isso mostra que existe uma potencialidade energética reprimida na semente de açaí que deve ser explorada adequadamente para geração de energia térmica na indústria para qualquer rendimento de caldeira.

É mostrado na tabela 4 resultados para a mistura dos combustíveis lenha e semente de açaí em várias proporções, pois a proposta do presente trabalho é também substituir gradativamente a lenha por semente de açaí.

TABELA 4 - Resultados do mix energético considerando diferentes proporções de combustíveis para um rendimento de caldeira de $50 \%$

\begin{tabular}{|c|c|c|c|c|}
\hline \multicolumn{4}{|c|}{ Quantidade (Kg/h) } & \multirow{2}{*}{$\begin{array}{l}\text { Entalpia de } \\
\text { saída do } \\
\text { vapor }(\mathrm{KJ} / \mathrm{Kg})\end{array}$} \\
\hline$\%$ & Semente de açaí & $\%$ & Lenha & \\
\hline 90 & 84,15 & 10 & 9,35 & 3403,31 \\
\hline 80 & 74,5 & 20 & 18,7 & 3316,45 \\
\hline 70 & 65,45 & 30 & 28,05 & 3229,59 \\
\hline 60 & 56,1 & 40 & 37,04 & 3142,73 \\
\hline 50 & 46,75 & 50 & 46,75 & 3055,83 \\
\hline 40 & 37,4 & 60 & 56,1 & 2968,97 \\
\hline 30 & 28,05 & 70 & 65,45 & 2882,11 \\
\hline 20 & 18,7 & 80 & 74,8 & 2795,20 \\
\hline 10 & 9,35 & 90 & 84,15 & 2708,34 \\
\hline
\end{tabular}

Para uma proporção de $90 \%$ de semente de açaí e $10 \%$ de lenha o valor da entalpia do vapor calculado é de $3403,31 \mathrm{KJ} / \mathrm{Kg}$, valor este, menor que a entalpia determinada para o vapor quando se pretende queimar somente semente de açaí, ou seja, 3490,21 KJ/kg (tabela 3). Nota-se também, nesta mesma tabela, que a diminuição da quantidade de semente no mix diminui a quantidade de energia liberada, ou seja, a semente de açaí determina uma maior quantidade de energia para o sistema. Vale salientar que, a proporção de $10 \%$ de semente de açaí e $90 \%$ de lenha determina uma entalpia de $2708,34 \mathrm{KJ} / \mathrm{kg}$, ou seja, maior ainda que o valor de $2621,48 \mathrm{KJ} / \mathrm{kg}$, calculado para a entalpia de saída do vapor quando a caldeira queima somente lenha.

O que determinará o mix ótimo serão as condições de operação do sistema energético e processo fabril, pois uma quantidade muito grande no mix de semente de açaí pode não trazer uma resposta operacional adequada, como exemplo muito entupimento dos tubos de fogo da caldeira flamotubular, embora, como já demonstrado o mix com sementes libera uma quantidade significativa de energia 
térmica. A maior liberação de energia pelas sementes de açaí, poderá proporcionar melhor controle dos equipamentos pasteurizadores e tanque de mistura, quando ocorrerem flutuações no processo e eventuais perdas de energia, que poderão ser compensadas pela maior quantidade de energia que está sendo disponibilizada.

Em termos ambientais, as vantagens na utilização de ambas as biomassas como combustíveis são as reduzidas emissões de poluentes, observadas nas composições químicas, com pouca quantidade de enxofre, que nas sementes de açaí são praticamente inexistentes comparadas à lenha, além disso os compostos de enxofre podem causar corrosão da chaminé. As emissões de $\mathrm{CO}_{2}$, liberadas durante a queima de biomassa, podem ser consideradas praticamente nulas, pois esse gás é reabsorvido no próximo ciclo de vida da planta, pelo processo de fotossíntese (SOUZA et al., 2015).

\section{CONCLUSÕES}

O potencial energético das sementes de açaí analisadas para um sistema queimando somente caroço de açaí e mix de caroço com lenha, em uma caldeira com 50 \% de eficiência térmica, apresentaram uma liberação de energia maior que a lenha utilizada na indústria em análise. Dessa forma, as sementes de açaí podem substituir totalmente a lenha como combustível da caldeira, e ainda auxiliar os pasteurizadores e tanque de mistura devido a maior necessidade de energia nesta etapa do processo.

Caso sejam aplicados procedimentos de manutenção para melhoria da eficiência da caldeira, progressivamente ocorre aumento do rendimento e maior liberação de energia para os pasteurizadores e tanque de mistura e consequentemente menor geração de subprodutos indesejáveis no processo de combustão. Os resultados também mostraram a grande quantidade de energia que pode ser extraida da semente de açaí, energia essa que pode ser utilizada na própria indústria que a gerou ou em outros sistemas energéticos.

Como recomendações sugere-se a realização do balanço exergético na caldeira, nos pasteurizadores e tanque de mistura para melhor entender as disponibilidades e irrverssibilidades energéticas que ocorrem nestes equipamentos. O procedimento de análise de umidade pode ser realizado no laboratório da própria indústria, para que se tenha um controle contínuo dessa característica do combustível. Sugere-se também que esse estudo seja aplicado em outros trabalhos que verifiquem a viabilidade do uso de outros tipos de sementes.

\section{REFERÊNCIAS}

ALMADA, T. S. de A.; SOUZA, M. J. R.; SANTANA, A. F. P.; GONÇALVES, R. F. B. Caracterização da casca do palmito para uso energético. Enciclopédia Biosfera Centro Científico Conhecer, v.14, n. 26, 2017. DOI: 10.18677/EnciBio_2017B13.

ÇENGEL, Y. A.; BOLES, M. A. Thermodynamics: an engineering approach. $8^{\circ}$ edition, McGraw Hill, 2015.

EMBRAPA. Sistemas de Produção 4 - Açaí. 2005. Disponível em:<http://ainfo.cnptia.embrapa.br/digital/bitstream/item/125409/1/SISTEMA-PROD4-ONLINE-.pdf>. Acesso em: set. 2016

FIGENER S/A - Engenheiros Associados. STEAM TABLE. São Paulo, 2000. 
LOO, S. V.; KOPPEJAN, J. The Handbook of Biomass Combustion and Cofiring. Earthscan, 2008.

NOBRE, J. R. C.; CASTRO, J. P.; BIANCHI, M. L.; BORGES, W. M. DA S.; TRUGILHO, P. F.; MOULIN, J. C. et al. Caracterização do carvão ativado produzido a partir de serragens de maçaranduba. Scientia Forestalis, v. 43, n. 107, p. 693702, 2015. Disponível em: < https://www.ipef.br/publicacoes/scientia/nr107/cap21.pdf >. Acesso em out 2016.

OLIVEIRA NETO, G. C. DE; FILHO, M. G.; GANGA, G. M. D.; NAAS, I. A.; VENDRAMETTO, O. Princípios e ferramentas da produção mais limpa: um estudo exploratório em empresas brasileiras. Gestão \& Produção, v. 22, n. 2, p. 326-344, 2015. http://dx.doi.org/10.1590/0104-530X1468-14

REMADE. Madeiras brasileiras e exóticas. Disponível em: $<$ http://www.remade.com.br/madeiras-exoticas/210/madeiras-brasileiras-e exoticas/macaranduba>. Acesso em: outubro. 2016.

RENDEIRO, G.; NOGUEIRA, M. F. M.; BRASIL, A. C. M.; CRUZ, D. O. de A.; GUERRA, D. R. da S. MACEDO, E. N.; ICHIHARA, J. A. Combustão e Gasificação de Biomassa Sólida. In: BARRETO, E. J. F. (Org.). Tecnologias de Energias Renováveis. Brasília: Ministério de Minas e Energia, 2008.

SANTANA, A. C.; CARVALHO, D. F.; MENDES, F. A. T. Organização e competitividade das empresas de polpas de frutas do Estado do Pará: 1995 a 2004. $1^{\circ}$ ed. Editora UNAMA, Universidade da Amazônia (UNAMA), 2006.

SANTOS, R. B.; LIMA, A. K. DE C. Análise comparativa do biogás: processo em biodigestores e de aterro sanitário. Revista Eletrônica de energia, v. 6, n. 1, p. 4857, 2016. https://revistas.unifacs.br/index.php/ree/article/download/4285/3062

SOUZA, V. H. A; dos SANTOS, L. T.; PAGEL, U. R.; SCARPATI, C. de B. L.; CAMPOS, A. F. Aspectos sustentáveis da biomassa como recurso energético, $\begin{array}{llllll}\text { Augustus, } & \text { v. } 20, \quad \text { n. } \quad 40, & \text { p. } & 105-123, & 2015 .\end{array}$ http://dx.doi.org/10.15202/19811896.2015v20n40p105

VAKKILAINEN, E. K. STEAM GENERATION FROM BIOMASS: Construction and Design of Large Boilers, $1^{\circ}$ edition, ELSEVIER, 2017. 